\title{
JEAN-ANTOINE DE BAÏF, Antigone
}

\section{Michele Mastroianni}

\section{(2) OpenEdition \\ Journals}

\section{Édition électronique}

URL : https://journals.openedition.org/studifrancesi/11707

DOI : 10.4000/studifrancesi. 11707

ISSN : 2427-5856

\section{Éditeur}

Rosenberg \& Sellier

\section{Édition imprimée}

Date de publication : 1 avril 2018

Pagination : 121-123

ISSN : 0039-2944

\section{Référence électronique}

Michele Mastroianni, « JEAn-AntoIne de baïF, Antigone », Studi Francesi [En ligne], 184 (LXII | I) | 2018, mis en ligne le 02 juillet 2018, consulté le 15 novembre 2021. URL : http://journals.openedition.org/ studifrancesi/11707 ; DOI : https://doi.org/10.4000/studifrancesi.11707

Ce document a été généré automatiquement le 15 novembre 2021.

\section{(c) (†)}

Studi Francesi è distribuita con Licenza Creative Commons Attribuzione - Non commerciale - Non opere derivate 4.0 Internazionale. 


\title{
JEAN-ANTOINE DE BAÏF, Antigone
}

\author{
Michele Mastroianni
}

\section{RÉFÉRENCE}

JEAN-ANTOINE DE BAïF, Antigone, édition par Monique Mund-Dopchie, dans CEuvres complètes, Paris, Champion, 2016, volume 2, pp. 5-133.

1 À travers une introduction qui pour être assez brève, n'en est pas moins efficace, Monique Mund-Dopchie encadre la pièce de Jean-Antoine de Baïf de manière rigoureuse, grâce à la discussion des problématiques majeures concernant la question de la traduction du modèle antique à la Renaissance, grec dans ce cas. En effet, à travers un paragraphe rapide (pp. 15-17) mais centré sur certaines des questions fondamentales de la réception du texte classique au xVI e siècle, notamment du texte tragique, l'éditeur moderne, spécialiste des réécritures d'Eschyle en France à l'heure de la Renaissance, focalise l'attention du lecteur sur deux éléments fondamentaux. Éléments fondamentaux au sein de la pratique de translatio au xvi siècle, tels que: 1) le respect du modèle ancien par une traduction littérale de l'œuvre source; 2) le respect du modèle antique, mais aussi la liberté poétique visant à l'originalité de la réécriture moderne, voire de la version en vernaculaire, donc, dans ce cas, du texte tragique, en version française, qui se pose forcément comme l'une des 'illustrations' possibles de la langue française, au sens d'un combat pour le soutien de la modernité et de l'exigence d'un nouveau genre tragique autochtone, en langue nationale. Donc, Monique MundDopchie introduit immédiatement son discours par une contextualisation historique et historiographique très efficace, déterminante pour comprendre le rôle du dramaturge français de l'époque (pas seulement celui de Jean-Antoine de Baïf et, en général, pas seulement celui du dramaturge français), à la fois vis-à-vis du texte classique ancien et des théorisations sur la langue moderne, évidemment liées, d'une part, au respect de la source ancienne, d'autre part, au dépassement du modèle. L'éditeur met ainsi l'accent sur une véritable et célèbre querelle qui - comme on le sait - s'accentue et s'approfondit au fil des discussions théoriques de la Renaissance, tout en étant déjà 
présente en pleine époque humaniste. De la sorte, Monique Mund-Dopchie rappelle aussi les avatars renaissants d'un débat sur la fidélité ou sur l'affranchissement du poète moderne par rapport à la source classique et quelques-uns des points essentiels d'un débat esthétique qui aboutira au XVII ${ }^{\mathrm{e}}$ siècle à la grande Querelle des Anciens et des Modernes, celle-là même qui passera encore au XVIII ${ }^{\mathrm{e}}$, au moins jusqu'au Théâtre des Grecs de Pierre Brumoy (1730). C'est la raison pour laquelle l'éditeur rappelle justement qu'au $\mathrm{XVI}^{\mathrm{e}}$ siècle «les théoriciens de la traduction [...] ne voulurent pas que l'opération se réalisât en violentant le français pour respecter l'original. C'est pourquoi ils furent d'accord, avec Étienne Dolet, pour exiger que le traducteur connût parfaitement la langue de l'auteur qu'il traduisait et la langue dans laquelle il le traduisait. Ils lui interdirent de rendre servilement le mot pour le mot, le vers pour le vers, la ligne pour la ligne, et l'invitèrent à faire preuve d'un art d'écrire. En revanche, ils ne furent pas unanimes à déterminer les limites de l'engagement du traducteur dans le travail littéraire qu'on exigeait de lui. On vit, d'un côté, Du Bellay proclamer l'impossibilité d'exprimer "le naïf d'une langue dans une autre" et ne reconnaître à la véritable traduction qu'une valeur pédagogique, ce qui ne l'empêcha pas, du reste, de revendiquer pour ses propres traductions la liberté créatrice. En revanche, Jacques Peletier du Mans prôna la soumission du traducteur au modèle et à la modération dans l'usage des néologismes; car le respect de l'œuvre originale exigeait, selon lui, une virtuosité propice au développement de la langue de la translation. Mais il ne fut guère suivi: à la fin $\mathrm{du} \mathrm{XvI}^{\mathrm{e}}$ siècle les traducteurs devinrent soit les serviteurs fidèles et méprisés des modèles antiques soit les rivaux des modèles qu'ils prétendaient désormais dépasser. Aux exigences de la versification française se superpose, selon Simone Maser et Alain Billault, une «volonté de réactualisation de la tragédie, non sans rapport avec la nostalgie éprouvée par Jean-Antoine de Baï pour une vocation de dramaturge qui n'a plus l'occasion de s'exprimer» (pp. 16-17). Certes, si d'une part, la question de la traduction, donc de la fidélité ou bien de l'infidélité du traducteur par rapport au modèle ancien est prioritaire, de même que l'actualisation ou la réactualisation au contexte moderne, dans une perspective sociale, politique, religieuse et esthétique (mais aussi au niveau linguistique et au niveau de la praxis concernant la réécriture en vernaculaire du modèle grec ou latin) - réactualisation forcément redevable au phénomène culturel du syncrétisme pagano-chrétien, central à la Renaissance - est tout à fait importante, d'autre part, il ne faut pas oublier (et peut-être aurait-il fallu insister encore sur cet aspect) que la notion de translatio, à plus forte raison si l'on considère la problématique des traductions de pièces tragiques classiques en France, est tout à fait liée à la notion d'inventio, donc aux réflexions théoriques - et aux modalités pratiques de les réaliser dans le texte moderne - concernant l'exigence de la part du traducteur d'opérer aussi en liberté, pour dépasser son modèle. Toutefois, il nous semble important de rappeler que s'il est vrai que l'humaniste de la Renaissance connaît la langue grecque, langue sur laquelle il opère d'habitude directement pour traduire et réécrire le texte d'origine (le texte tragique dans ce cas), il est vrai de même que dans plusieurs circonstances l'impossibilité ou l'incapacité de comprendre la langue grecque, en particulier celles des chœurs, empêche malgré lui le dramaturge moderne de rester fidèle à sa source. Il est ainsi évident que dans ce cas la non compréhension du texte tragique de départ pousse normalement le traducteur humaniste vers l'amplificatio de son modèle, donc vers des glissements de sens et comme dans le cas de Calvy de La Fontaine (L'Antigone de Sophoclés, 1542), pour en rester strictement au sujet des réécritures du mythe d'Antigone en France au XVI ${ }^{e}$ siècle - vers 
la construction de véritables sections qui déplacent la réflexion, du contexte politique au contexte théologique, dans la direction d'une confession et d'une herméneutique catholique ou protestante. Ce qui met même en cause - ainsi que Monique MundDopchie le souligne très bien - le problème de la médiation du texte antique à la Renaissance. Problème ou réalité poétique qui explique aussi les phénomènes de changements sémantiques et de déplacements idéologiques d'un texte à l'autre (de l'ancien au moderne), problème qui dans certains cas, comme dans celui de l'Antigone de Calvy de La Fontaine, reste ouvert étant donné que la traduction en prose dont il déclare s'être servi, encore aujourd'hui n'a pas été retrouvée. Dans ce cas, donc, il n'est pas possible de dire, par exemple, si les importants glissements de sens de la traduction de Calvy, en particulier dans la direction d'une relecture catholique de l'héroïne éponyme et de l'interpretatio de la loi divine par rapport à la loi terrestre, soient dus à la version en prose perdue, dont Calvy parle dans la pièce liminaire, Au lecteur salut, de sa tragédie (cf. Calvy de La Fontaine, L'Antigone de Sophoclés, edizione critica con commento a cura di M. Mastroianni, Alessandria, Edizioni dell'Orso, 2000, p. 15) ou plutôt à une volonté précise de la part de cet auteur de relire la tragédie de Sophocle à la lumière de notions catholiques, telles que celle de charité ou de peché. Mais au de-là de ces aspects, Monique Mund-Dopchie réfléchit encore sur un point saillant concernant la lecture du texte grec sophocléen de la part de Jean-Antoine de Baïf, et elle se pose le problème de l'édition utilisée par le traducteur français («comme notre poète connaissait manifestement le grec, il a très certainement eu accès à l'original, sans passer par la médiation exclusive d'une traduction latine. Mais à travers quelle édition?», p. 14). Problème que Monique Mund-Dopchie essaie de circonscrire en rappelant que «les seules certitudes dont nous disposons pour le moment concernent la famille des manuscrits à laquelle le texte de Baïf s'apparente. Plusieurs indices penchent, en effet, en faveur de la tradition représentée par le Parisinus graecus 2712, daté du XIII siècle: à l'instar de ce manuscrit, le texte de Baïf attribue au messager et non au garde les vers 384-385, à Ismène et non au chœur le vers 576; de même, il adopte au vers 53 la leçon pathos (ô malheurté doublee) au lieu d'epos (ce double nom), au vers 352 la leçon admēta (toreau indomté) au lieu d'akmēta (infatigable), au vers 514 la leçon dussebei (à ce méchant) au lieu de dussebē (honneurs impies)» (p. 15). Du reste, souligne Monique Mund-Dopchie, Jean-Antoine de Baïf «disposait, en se cantonnant à la France, de trois éditions du texte grec et de trois traductions latines imprimées, sans compter les travaux d'Henri Estienne, publiés à Genève» (p. 14). Or, de la question éditoriale du texte grec probablement lu par Jean-Antoine de Baïf elle passe, à travers une analyse ponctuelle, à une réflexion sur la versification de la tragédie française (pp. 30-33) et à un bilan final concernant le texte de Baïf, que justement Mund-Dopchie définit comme une «adaptation culturelle» au public et au contexte politique, et pas seulement politique, de la France de l'époque, en concluant que «l'Antigone de Baiff est donc bien une traduction de la tragédie de Sophocle, dont elle se sert comme modèle à trois exceptions près. Elle s'efforce d'en être le miroir fidèle, dans les limites qu'imposent toutefois, d'une part, les contraintes imposées par le recours aux vers rimés, d'autre part, la prégnance des goûts d'un public mondain et peut-être un certain souci dramaturgique» (p. 29).

2 C'est ainsi que Monique Mund-Dopchie fait paraître pour les éditions Champion, en édition séparée par rapport aux Jeux (trois volumes), «ceux-ci formant eux-mêmes le tome III de l'édition des CEuvres en rimes» (p. 7), œuvres dont l'édition monumentale est dirigée par Jean Vignes, une édition critique excellente, publiée dans le volume des Jeux 
(1572-1573). Le texte a été reproduit dans l'édition de Monique Mund-Dopchie selon le principe de la conservation de l'orthographe et de la ponctuation de l'édition du XVI siècle (1572). Un dossier critique rigoureux et très utile pour les spécialistes de la tragédie renaissante, mais aussi pour les étudiants universitaires (pp. 103-122), accompagne ce travail qui se clôt par un glossaire, un index des noms propres cités dans l'introduction et un index des noms de personnes et des divinités citées dans la tragédie. 